\title{
Editorial: Unifying Ecology Across Scales: Progress, Challenges and Opportunities
}

\author{
Mary I. O'Connor ${ }^{1 *}$, Diego R. Barneche ${ }^{2,3}$, Angélica L. González ${ }^{4}$ and Julie Messier ${ }^{5}$ \\ ${ }^{1}$ Department of Zoology and Biodiversity Research Centre, University of British Columbia, Vancouver, BC, Canada, \\ ${ }^{2}$ Australian Institute of Marine Science, Crawley, WA, Australia, ${ }^{3}$ Oceans Institute, University of Western Australia, Crawley, \\ WA, Australia, ${ }^{4}$ Department of Biology, Center for Computational and Integrative Biology, Rutgers University, Camden, NJ, \\ United States, ${ }^{5}$ Biology Department, University of Waterloo, Waterloo, ON, Canada
}

Keywords: ecology, global change, models, allometry, information theory, metabolic theory of ecology (MTE), population dynamics

Editorial on the Research Topic

Unifying Ecology Across Scales: Progress, Challenges and Opportunities

\section{THE NEED TO UNIFY ECOLOGY: A GRAND CHALLENGE FOR A CHANGING WORLD}

OPEN ACCESS

Edited and reviewed by: Peter Convey, British Antarctic Survey (BAS), United Kingdom

${ }^{*}$ Correspondence:

Mary I. O'Connor

oconnor@zoology.ubc.ca

Specialty section:

This article was submitted to Biogeography and Macroecology,

a section of the journal

Frontiers in Ecology and Evolution

Received: 25 September 2020 Accepted: 09 October 2020

Published: 30 October 2020

Citation:

O'Connor MI, Barneche DR,

González AL and Messier J (2020) Editorial: Unifying Ecology Across Scales: Progress, Challenges and

Opportunities.

Front. Ecol. Evol. 8:610459.

doi: 10.3389/fevo.2020.610459
Ecology is the science of how living systems grow, change, and persist. Although this is not the definition presented in most textbooks, this is the central theme of this scientific discipline as it is practiced in the current era of rapid global change. Change comes in many forms, from natural succession of communities to biological invasions affecting patterns of biodiversity to the collapse of fisheries. Understanding and forecasting natural change as well as mitigating undesirable anthropogenic change is one of the grand challenges we face in the twenty-first century.

With our collective focus on global change, the ecological sciences-from organismal, population, community, and ecosystem ecology to evolutionary ecology-are undergoing a revolution. It has become clear that although we have vast and multifaceted ecological knowledge, it has yet to solidify into a coherent body of science. For example, over decades in the latter half of the twentieth century researchers, journals and even entire academic departments focused on specific branches of ecological thinking as narrowly defined as behavioral ecology, population ecology, or community ecology. Currently though, it has become clear that to understand our changing world and our place in it, examining the ecological changes afoot from a fragmented and narrow disciplinary perspective is insufficient. Population dynamics, ecosystem functions, individual behavior, and other aspects of living systems are deeply connected, and we cannot project changes in one without understanding how they are related to other processes across scales of space and time and levels of biological organization.

\section{CONTRIBUTIONS IN THIS SPECIAL TOPIC}

In recent decades, efforts to unify ecological understanding across scales and levels of organization have flourished and disciplinary barriers have fallen (see, for example, Brown et al., 2004; Scheiner and Willig, 2008; Harte, 2011; Marquet et al., 2014). In this special topic, we highlight recent progress in the unification of ecological sciences. Papers in this issue can be organized in three groups. Some papers take a very broad view on the current state of ecology, proposing frameworks and approaches to organize understanding and guide new research (Fulton et al.; Hodapp et al.; 
Kempes et al.; Martinez; O'Connor et al.). A second group provides concrete tools and approaches to unifying ecological data and models, through specific and quantitative frameworks (McCabe and Dietze; Newman et al.; Niklas and Hammond; Tekin et al.; Yen et al.). A third group provides specific analyses that reveal new insights into how dynamics in natural systems are linked across scales and levels of organization (Amarasekare; Bideault et al.; Cavan et al.; Gibert and Yeakel; Pawar et al.; Segura and Perera; Zelnik et al.; Ziv and Davidowitz). Together, this issue is full of big ideas complemented by specific analyses, providing an exciting and welcome step in our ongoing effort for a unified science of ecology.

Fulton et al. offer a sweeping view of ecology, moving through what they identify as a transitional period now to a future in which we will have developed a more coherent ecological science through better models. This paper is a mustread, providing an impressive and inspiring perspective on the successes of synthesis in ecology through models. Among the many successes they highlight is the recognition that we understand that human activities and evolutionary change both occur within ecological time scales, and "this new reality drives home that ecology lacks a universally solid foundation from which to extend existing theories and modeling approaches to easily absorb the many interacting components and scales." Fulton et al. identify immediate challenges as the need to embrace new modeling approaches that span more dimensions and allow more dynamic and evolving parameterizations and structures to allow models to incorporate new sources of data. They emphasize that these advances require not only technical advances in modeling, but also coordinated advances in ecological theory and concepts, as well as multiscale data streams. To continue the ecological tradition of using models to explain and predict, now in a rapidly changing world, they argue for continued investment in an approach that unifies disparate ideas while also allowing multiple approaches to be employed to solve the problems we face.

Other contributions propose frameworks to unify concepts across scales, disciplines, and levels of organization. Consistent with Fulton et al.'s call for greater attention to a universally solid foundation for ecological science, O'Connor et al. propose strengthening the foundation by integrating concepts of information and information theory into ecology's fundamental principles. They review major concepts in information theory, and highlight how these fundamental principles of science have not been fully explored in ecological understanding. Their paper provides a set of ecological principles grounded firmly in a science that includes information processing as fundamental to living systems, along with energy flows and material cycles. Similarly, with firm grounding in these first principles, Kempes et al. provide scaffolding that explains how evolution must occur within the boundaries of the physical world, and what these physical constraints imply for all that we still do not know about evolution. Martinez extends a unifying framework further with allometric networks, and like several other proposed frameworks in this issue, Martinez includes humans and socioecological systems in his unifying scheme. Hodapp et al. provide additional structure through emphasizing resource use efficiency as a unifying concept across diverse living systems, which may differ in the identity and number of limiting resources, trophic levels, and ecological, temporal and spatial scales.

Inspired by the broad roadmap laid out by contributions like those described above, we might then ask, "how do we proceed and implement a unifying framework?". This is a hard question. Thoughtful and constructive answers are provided in every paper in this edition. Yen et al. identify the statistical challenge of integrating data-often collected specifically at one level of biological organization-across multiple levels of organization. They provide an approach integrating multiple data types and parameterize more complex, process-explicit models, providing a path forward to testing multi-scale theory using data spanning many organizational levels. Niklas and Hammond's contribution dives into understanding body form and function in generalized terms, and renders the allometric scaling model more biologically relevant and useful by exposing the value and meaning of the often-overlooked scaling normalization constant. Newman et al. identify and explain specific challenges that we face when trying to understand emergent patterns of complex ecological systems at landscape scales: coarse-graining, the middle number problem of the domain of data complexity, and non-stationarity issues when predicting future ecological states from models based on adjustable parameters. McCabe and Dietze offer a solution to another landscape-scale problem-scaling contagious disturbance. They provide an approach that allows modeling of such events in the context of climate change, thereby linking community-scale disturbance events with broader scale climate change and feedbacks. Cavan et al. also work with climate change models and finer scale ecological dynamics by using the metabolic theory of ecology (MTE) to link rates of carbon flux in microbial systems to global climate feedbacks.

Tekin et al.'s contribution provides an approach that could potentially also serve as an important part of a unifying framework by demonstrating that many if not all measures of pairwise interactions-present in a diverse array of complex systems across very different scientific disciplines-can be derived from and analyzed in the context of a single mathematical framework. They build upon this finding to introduce consistent measures of higher order interactions. In this way they provide a practical tool and an insightful guide to thinking about complexity that is likely to benefit unification efforts across ecological (and other scientific) disciplines.

The third set of papers in this issue dives deeper into ecological and evolutionary dynamical models. These contributions implement unifying concepts by integrating ideas from diverse areas of thought into a single framework for population dynamics, and then analyzing the models to understand possible patterns and processes in nature. Gibert and Yeakel demonstrate how evolutionary change within the context of ecological dynamics can provide unexpected links between individual traits and the structure of an emergent food web. Ziv and Davidowitz link landscape-scale features (patch size and fragmentation) to evolutionary outcomes (selection for life-history traits increasing survival) and population dynamics (likelihood of population extinction). The need for strong quantitative general predictions of fluctuations of species abundance are addressed by Segura and Perera through deriving a theoretical model 
that links metabolism to abundance fluctuations in ecological populations and communities. Amarasekare carefully considers how physiological effects of temperature on the rates that drive consumer resource dynamics can lead to very different consequences of warming and climate change in different thermal regimes (e.g., tropics vs. temperature). Tackling the same question from a different angle, Bideault et al. find that the way temperature dependences are distributed among the processes that determine consumer-resource interactions and outcomes can substantially affect the ultimate outcome of warming. Pawar et al. build on an immense database of consumer-resource interactions and related traits to observe that body size distributions differ for consumers foraging in two-dimensional and three-dimensional habitats. They provide a theoretical explanation for this pattern, and use their joint mathematical/empirical approach to offer new insights about how consumer resource interactions are constrained and how they flourish in different environments. Zelnik et al. provide a theoretical analysis of the effects of different spatial and temporal properties of disturbance on ecosystem stability. Their modeling approach provides insights into the tight link between temporal and spatial dimensions on ecosystem responses to disturbance. By integrating Earth System Models and satellitederived temperature data, and using principles from MTE, Cavan et al. estimate changes in global exports of marine carbon. Their findings suggest a main role of temperature sensitivity shaping carbon outputs and call for the need of incorporating organismal temperature dependence into biogeochemical models. Each of these contributions is exciting, novel and potentially transformative to how we think about ecological dynamics and the structures they produce. These are the bricks and mortar of a unified science, providing specific and clear

\section{REFERENCES}

Brown, J. H., Gillooly, J. F., Allen, A. P., Savage, V. M., and West, G. B. (2004). Toward a metabolic theory of ecology. Ecology 85, 1771-1789. doi: $10.1890 / 03-9000$

Harte, J. (2011). Maximum Entropy and Ecology, a Theory of Abundance, Distribution and Energetics. Oxford, NY: Oxford University Publishing.

Marquet, P. A., Allen, A. P., Brown, J. H., Dunne, J. A., Enquist, B. J., Gillooly, J. F., et al. (2014). On theory in ecology. Bioscience 64, 701-710. doi: 10.1093/biosci/biu098

Scheiner, S. M., and Willig, M. R. (2008). A general theory of ecology. Theor. Ecol. 1, 21-28. doi: 10.1007/s12080-007-0002-0 new ideas and observations to our body of knowledge. Many work at the intersection between population dynamics and the unifying approach of MTE, which is based on general principles of how ecological rates of energy flux and material cycling scale with body size and temperature (Brown et al., 2004).

The contributions in this issue advance current themes in unifying efforts, specifically those employing MTE (Bideault et al.; Cavan et al.) or its core allometric scaling model (Kempes et al.; Martinez; Niklas and Hammond), those integrating data and models (Fulton et al.; Newman et al.; Yen et al.), bridging between local scale dynamics and climate change models (Cavan et al.; Fulton et al.; McCabe and Dietze), and those focusing on resource use and trade-offs (Hodapp et al.; Ziv and Davidowicz). Newer themes in unifying ecological science are also emerging, in the form of emphasis on disturbance and its multi-scale consequences (McCabe and Dietze; Segura and Perera; Zelnik et al.), on trait based perspectives on consumer-resource interactions and interaction strengths (Amarasekare; Bideault et al.; Pawar et al.; Tekin et al.), eco-evolutionary feedbacks (Gilbert and Yeakel; Kempes et al.) and on information as a unifying principle for mainstream ecological thinking (O'Connor et al.). Together, the full issue opens doors and guides our steps through them in our collective efforts to better understand our changing biosphere through ecological science.

\section{AUTHOR CONTRIBUTIONS}

All authors created the vision for the special issue, served as editors, and discussed the main concept for the editorial manuscript. MO'C wrote the manuscript. All authors reviewed and edited it.
Conflict of Interest: The authors declare that the research was conducted in the absence of any commercial or financial relationships that could be construed as a potential conflict of interest.

Copyright (C) 2020 O'Connor, Barneche, González and Messier. This is an open-access article distributed under the terms of the Creative Commons Attribution License (CC $B Y)$. The use, distribution or reproduction in other forums is permitted, provided the original author(s) and the copyright owner(s) are credited and that the original publication in this journal is cited, in accordance with accepted academic practice. No use, distribution or reproduction is permitted which does not comply with these terms. 